\title{
Post-transcriptional regulation of tumour necrosis factor $\alpha$ production
}

\author{
Paul Anderson
}

Tumour necrosis factor $\alpha(\mathrm{TNF} \alpha)$ is a proinflammatory cytokine produced by activated macrophages, lymphocytes and other cells. $^{1-3}$ The production of TNF $\alpha$ is under transcriptional and post-transcriptional control. ${ }^{4-10}$ Post-transcriptional control of $\mathrm{TNF} \alpha$ expression is achieved by regulating translational initiation, mRNA stability, and polyadenylation. ${ }^{6710} \mathrm{An}$ adenine and uridine (AU)-rich element (ARE) in the 3' untranslated region (3' UTR) of TNF $\alpha$ transcripts ${ }^{11-13}$ is an important determinant of posttranscriptional control. Transfer of this element to heterologous reporter transcripts changes the expression of the reporter protein. Transacting factors that bind to the ARE and participate in post-transcriptional control have recently been identified. The ARE binding proteins expressed in a given cell are thought to determine the level of protein expression.

\section{AU-rich elements (AREs)}

The 3' untranslated region of mRNAs encoding short lived immediate early genes (for example, fos, jun) as well as selected cytokines (for example, TNF $\alpha, \mathrm{GM}-\mathrm{CSF}$ ) possess AUrich elements that regulate protein expression. Class I AREs consist of one or more pentamer repeats (that is, AUUUA), whereas class II AREs consist of one or more nonamer repeats (that is, AUUUAUUUA). Whereas class I AREs are sufficient to promote the degradation of transcripts encoding immediate early proteins, both class I and class II AREs regulate the production of cytokines such as $\mathrm{TNF} \alpha$, GM-CSF, interleukin 3 (IL3) and interferon $\alpha$ (IFN $\alpha$ ). The 3' untranslated region of $\mathrm{TNF} \alpha$ transcripts contains both class I and class II AREs. Electrophoretic mobility shift assays have identified two distinct regulatory complexes that assemble on these adjacent cis elements in HeLa cells. ${ }^{14}{ }^{15}$ The complex assembled on the class II ARE (designated complex 1) includes the related RNA binding proteins TIA- 1 and TIAR. ${ }^{14}$ The complex assembled on the class I ARE (designated complex 2) includes an unidentified $55 \mathrm{kDa}$ protein. Whereas complex 1 forms with extracts derived from activated and unactivated macrophages, complex 2 is only formed using extracts derived from activated macrophages.

The importance of the TNF $\alpha$ ARE is underscored by the severe phenotype of transgenic knock-in mice expressing $\mathrm{TNF} \alpha$ transcripts that lack the ARE. ${ }^{16}{ }^{17}$ Overexpression of TNF $\alpha$ by these animals results in chronic inflammatory arthritis and inflammatory bowel disease. The overexpression of $\mathrm{TNF} \alpha$ is a consequence of increased transcript stability combined with a loss of translational silencing. Furthermore, the inhibition of $\mathrm{TNF} \alpha$ production by cytokine suppressive anti-inflammatory drugs (CSAIDs) was absent in these animals, indicating that the TNF $\alpha$ ARE mediates the translational silencing that is abrogated by inhibition of p38 MAP kinase. As a result of these defects, synovial fibroblasts spontaneously produce $\mathrm{TNF} \alpha$ in the mutant mouse.

\section{ARE binding proteins}

Transacting factors that bind to the TNF $\alpha$ ARE are essential for post-transcriptional control of TNF $\alpha$ expression. For example, the zinc-finger protein tristetraprolin (TTP) binds to the TNF $\alpha$ ARE and promotes the degradation of TNF $\alpha$ transcripts. ${ }^{18-20}$ Mutant mice lacking TTP develop cachexia, arthritis and autoimmunity as a consequence of overexpressed $\mathrm{TNF} \alpha \mathrm{mRNA}$ and protein. ${ }^{20}$ This clinical syndrome can be prevented by the repeated injection of neutralising antibodies reactive with $\mathrm{TNF} \alpha .{ }^{20}$ In addition to TTP, the RNA binding proteins Hel-N $1,{ }^{21} \mathrm{HuR}^{22-25}$ AUF1 (hnRNP D), ${ }^{26}$ and TIAR $^{14}$ are ARE binding proteins that have been proposed to regulate the expression of $\mathrm{TNF} \alpha{ }^{27} \mathrm{Hel}-$ $\mathrm{N}^{212829}$ and HuR ${ }^{22-25}$ stabilise ARE containing transcripts, whereas AUF1 destabilises these same transcripts. ${ }^{30}$ It is therefore likely that the stability of ARE containing transcripts is determined by the relative expression of functionally antagonistic ARE binding proteins.

\section{TIA-1 and TIAR}

TIAR and its closely related homologue TIA-1 are members of the RNA recognition motif (RRM) family of RNA binding proteins. ${ }^{31-35}$ Both proteins possess three RRM domains at their amino termini that confer high affinity binding to uridine-rich motifs. ${ }^{32}$ Like components of the general heteronuclear ribonucleoprotein complex (hnRNP), TIA-1 and TIAR continuously shuttle between the nucleus and the cytoplasm (N Kedersha and P Anderson, manuscript in preparation), suggesting that they might participate in the nucleocytoplasmic transport of selected mRNAs. These proteins also regulate the general translational arrest that accompanies environmental stress. After the stress induced phosphorylation of translation initiation factor eIF-2 $\alpha$, TIA- 1 and TIAR recruit most cytoplasmic mRNAs to discrete foci known as stress granules. ${ }^{36}$ The TIA$1 / \mathrm{R}$ dependent sequestration of these mRNAs prevents their translational initiation. In this capacity, TIA-1 and TIAR function as translational silencers that seem to influence the duration of stress induced translational arrest. 
Mutant mice lacking TIAR exhibit partial embryonic lethality and defective germ cell maturation, implicating this protein in selective aspects of vertebrate development. ${ }^{37}$

The discovery of TIAR as a component of the ARE associated complex that assembles on the 3' UTR of TNF $\alpha$ transcripts ${ }^{14}$ provided the first clue that TIA-1 and TIAR might specifically regulate the expression of $T N F \alpha$. To test this hypothesis, we produced mutant mice lacking TIA-1 and compared the LPS induced expression of TNF $\alpha$ in wild type and TIA-1-/macrophages. ${ }^{38}$ Our results indicate that LPS induced expression of $\mathrm{TNF} \alpha$ is significantly increased in macrophages lacking TIA $-1 .^{38}$ The functional effects of TIA-1 seem to result from translational silencing rather than regulation of mRNA stability. ${ }^{38}$ Thus, the ARE binding protein TIA-1 represses the expression of TNF $\alpha$ by a mechanism that differs from that used by other known ARE binding proteins.

Although both TIA-1 and TIAR are concentrated in the nucleus at steady state, heterokaryon analysis shows that both proteins continuously shuttle between the nucleus and the cytoplasm (Kedersha and Anderson, manuscript in preparation). In this respect, TIA-1 and TIAR resemble the heteronuclear ribonucleoproteins (hnRNPs) that assemble around nascent RNA transcripts and facilitate transport from the nucleus to the cytoplasm. ${ }^{39}$ Like the hnRNPs, TIA-1 and TIAR can function as general RNA binding proteins that interact with many, if not most, mRNAs in vitro. ${ }^{32} \mathrm{At}$ the same time, these proteins can selectively interact with RNAs possessing uridine-rich motifs. ${ }^{32}$ In their ability to function as both general and specific RNA binding proteins, TIA- 1 and TIAR resemble hnRNPs K and E1, proteins that participate in general RNA export and also bind to the 3' UTRs of 5- lipoxygenase transcripts to repress translational initiation..$^{41}$

\section{Conclusions}

Our results introduce TIA-1 and TIAR as translational silencers that can independently and selectively regulate the production of $\mathrm{TNF} \alpha$. Previous studies using macrophage cell lines have clearly shown that translational silencing is important in the posttranscriptional control of $\mathrm{TNF} \alpha$ production. ${ }^{4}$ 710 In the unstimulated macrophage cell line RAW 264.7, $\mathrm{TNF} \alpha$ transcripts are expressed but excluded from polysomes and not translated. ${ }^{4}{ }^{6}$ Comparison of $\mathrm{TNF} \alpha \mathrm{mRNA}$ distribution into polysomes in wild type and TIA-1-/- macrophages indicate that TIA-1 controls the association of TNF $\alpha$ mRNA with polysomes. It remains to be determined whether TIA-1/R induced translational silencing is achieved by regulation of translational initiation. In any case, the ability of TIA-1 and TIAR to inhibit TNF $\alpha$ mRNA translation suggests that these proteins might be targets of the stress kinase signalling cascade that is blocked by CSAIDS. CSAIDS block the LPS induced production of $\mathrm{TNF} \alpha$ by preventing translational de-repression. ${ }^{82}$ This is accomplished by inhibiting the p38-MK2 signalling cascade, ${ }^{43-45}$ suggesting that these kinases phosphorylate a translational silencer that associates with TNF $\alpha$ transcripts. The ability of CSAIDS to similarly repress the expression of $\mathrm{TNF} \alpha$ in wild type and TIA-1-/- macrophages indicates that TIA-1 is not an essential target of these drugs. TIA-1 might thus act as a constitutive translational suppressor controlling excessive $\mathrm{TNF} \alpha$ production. Alternatively, the functional redundance of TIA-1 and TIAR leaves open the possibility that cells lacking both TIA-1 and TIAR might be resistant to the suppressive effects of CSAIDS.

Taken together, our results suggest that TIA-1 and TIAR are translational silencers that regulate the cellular and organismal response to stress. At the cellular level, these proteins contribute to the general translational arrest that accompanies environmental stress. By controlling the duration of translational arrest, TIA-1 and TIAR might determine whether stressed cells live to repair the stress induced damage or die by apoptosis. At the organismal level, these proteins regulate the expression of at least one inflammatory mediator that serves as a sentinel to signal the presence of microbial infection. It remains to be determined whether the translational control exerted by these proteins is limited to the stress response, or is a general feature of normal cellular metabolism.

1 Beutler B. The role of tumor necrosis factor in health and disease. J Rheumatol 1999;26: 16-21.

2 Feldmann M, Maini R. The role of cytokines in the pathogenesis of rheumatoid arthritis. Rheumatology 1999;38: $-7$

3 Kollias G, Douni E, Kassiotis G, et al. The function of TNF and receptors in models of multi-organ inflammation, heumatoid arthritis, multiple scleroisis and inflammatory bowel disease. Ann Rheum Dis 1999;58:I32-9.

4 Crawford EK, Ensor JE, Kalvakolanu I, et al. The role of 3' poly (A) tail metabolism in tumor necrosis factor- $\alpha$ regulation. J Biol Chem 1997;272:21120-7.

5 Biragyn A, Nedospasov SA. Lipopolysaccharide-induced expression of TNF- $\alpha$ gene in the macrophage cell line ANA-1 is regulated at the level of transcription processivity. J Immunol 1995;155:674-83.

6 Han J, Brown T, Beutler B. Endotoxin-responsive sequences control cachectin/tumor necrosis factor biosynthesis at the control cachectin/tumor necrosis factor biosynth

7 Han J, Beutler B. The essential role of the UA-rich sequence in endotoxin-induced cachectin/TNF synthesis. Eur Cytokine Netw 1990;1:71-5.

8 Prichett W, Hand A, Shields J, et al. Mechanism of action of bicyclic imidazoles defines a translational regulatory pathway for tumor necrosis factor alpha. J Inflamm 1995;45:97-105.

9 Raabe T, Bukrinsky M, Currie RA. Relative contribution of transcription and translation to the induction of tumor necrosis factor- $\alpha$ by lipopolysaccharide. J Biol Chem 1998; 273:974-80

10 Wang E, Ma WJ, Aghajaniam C, et al. Posttranscriptional regulation of protein expression in human epithelial carcinoma cells by adenine-uridine-rich elements in the 3'-untranslated region of tumor necrosis factor- $\alpha$ messenger RNA. Cancer Res 1997;57:5426-33.

11 Caput D, Beutler B, Hartog K, et al. Identification of a common nucleotide sequence in the 3'-untranslated region of mon nucleotide sequence in the 3'-untranslated region of Natl Acad Sci USA 1986;83:1670-4.

12 Kruys V, Marinz O, Shaw G, et al. Translational blockade mposed by cytokine-derived UA-rich sequences. Science 1989;245:852-5.

13 Lewis T, Gueydan C, Huez G, et al. Mapping of a minimal AU-rich sequence required for lipopolysaccharide-induced binding of a $55-\mathrm{kDa}$ protein on tumor necrosis factor- $\alpha$ mRNA. J Biol Chem 1998;273: 13781-6.

14 Gueydan C, Droogmans L, Chalon P, et al. Identification of TIAR as a protein binding to the translational regulatory AU-rich element of tumor necrosis factor $\alpha$ mRNA. J Biol Chem 1999;274:2322-6.

15 Hel Z, Skamene E, Radzioch D. Two distinct regions in the 3' untranslated region of tumor necrosis factor alpha mRNA form complexes with macrophage proteins. Mol Cell Biol 1996;16:5579-90.

16 Kontoyiannis D, Pasparakism M, Pizarro TT, et al. Impaired Kontoyiannis D, Pasparakism M, Pizarro TT, et al. Impaired
on/off regulation of TNF biosynthesis in mice lacking TNF 
AU-rich elements: implications for joint and gut-associated immunopathologies. Immunity 1999;10:387-98.

17 Keffer J, Probert Lazlaris H, et al. Transgenic mice expressing human tumour necrosis factor: a predictive genetic model of arthritis. EMBO J 1991;10:4025-31.

18 Carballo E, Lai WS, Blackshear PJ. Feedback inhibition of macrophage tumor necrosis factor- $\alpha$ production by tristetraprolin. Science 1998;281:1001-5.

19 Lai WS, Carballo E, Strum JR, et al. Evidence that tristetraprolin binds to AU-rich elements and promotes the deadenylation and destabilization of tumor necrosis factor alpha mRNA. Mol Cell Biol 1999;19: 4311-23.

20 Taylor GA, Carballo E, Lee DM, et al. A pathogenetic role for TNF $\alpha$ in the syndrome of cachexia, arthritis, and autoimmunity resulting from tristetraprolin (TTP) deficiency. Immunity 1996;4:445-54.

21 Levine TD, Gao F, King PH, et al. Hel-N1: an autoimmune RNA-binding protein with specificity for 3' uridylate-rich untranslated regions of growth factor mRNAs. Mol Cell Biol 1993;13:3494-504.

22 Fan XC, Steitz JA. Overexpression of HuR, a nuclearcytoplasmic shuttling protein, increases the in vivo stability of ARE-containing mRNAs. EMBO J 1998;17:3448-60.

23 Myer VE, Fan XC, Steitz JA. Identification of HuR as a protein implicated in AUUUA-mediated mRNA decay. EMBO J 1997;16:2130-9.

24 Peng SSY, Chen CYA, Xu N, et al. RNA stabilization by the AU-rich element binding protein, HuR, an ELAV protein. EMBO J 1998; 17:3461-70.

25 Sokolowski M, Furneaux H, Schwartz S. The inhibitory activity of the AU-rich RNA element in the human papillomavirus type 1 late 3' untranslated region correlates with its affinity for the elav-Like HuR protein. J Virol 1999;73: 1080-91.

26 Zhang W, Wagner BJ, Ehrenman K, et al. Purification, characterization, and cDNA cloning of an AU-rich element RNA-binding protein, AUF1. Mol Cell Biol 1993;13: 7652-65.

27 Sakai K, Kitagawa Y, Hirose G. Binding of neuronal ELAVlike proteins to the uridine-rich sequence in the 3'like proteins to the uridine-rich sequence in the 3 untranslated region of tumor necrosis factor

28 Gao FB, Carson CC, Levine TD, et al. Selection of a subset Gao FB, Carson CC, Levine TD, et al. Selection of a subset of mRNAs from combinatorial 3' untranslated region libraries using neuronal RNA-binding protein

29 Ford LP, Watson J, Keene JD, et al. ELAV proteins stabilize deadenylated intermediates in a novel in vitro mRNA deadenylation/degradation system. Genes Dev 1999;13. $188-201$

30 Loflin P, Chen CYA, Shyu AB. Unraveling a cytoplasmic role for hnRNP D in the in vivo mRNA destabilization directed by the AU-rich element. Genes Dev 1999;13: 1884-97.
31 Kawakami A, Tian O, Dvam X, et al. Identification and functional characterization of a TIA-1-related nucleolysin. Proc Natl Acad Sci USA 1992;89:8681-5.

32 Dember LM, Kim ND, Liu KQ, et al. Individual RNA recognition motifs of TIA-1 and TIAR have different RNA binding specificities. J Biol Chem 1996;271:2783-8.

33 Beck ARP, Medley QG, O'Brien S, et al. Structure, tissue distribution and genomic organization of the murine RRM-type RNA binding proteins TIA-1 and TIAR. Nucleic Acids Res 1996;24:3829-35.

34 Kawakami A, Tian Q, Strenli $\mathrm{M}$, et al. Intron-exon organization and chromosomal localization of the human TIA-1 gene. J Immunol 1994;152:4937-45

35 Tian $\mathrm{Q}$, Strenli $\mathrm{M}$, Saito $\mathrm{H}$, et al. A polyadenylate binding protein localized to the granules of cytolytic lymphocytes induces DNA fragmentation in targetcells. Cell 1991;67: 629-39.

36 Kedersha NL, Gupta M, Li W, et al. RNA-binding proteins TIA- 1 and TIAR link the phosphorylation of eIF- $2 \alpha$ to the assembly of mammalian stress granules. J Cell Biol 1999;47:1431-41.

37 Beck ARP, Miller IJ, Anderson P, et al. RNA-binding protein TIAR is essential for primordial germ cell development. Proc Natl Acad Sci USA 1998;95:2331-6.

38 Piecyk M, Wax S, Beck ARD, et al. TIA-1 is a translational silencer that selectively regulates the expression of TNfalpha. EMBO J 2000;19:4154-63.

39 Krecic A, Swanson M. hnRNP complexes: composition, structure and function. Curr Opin Cell Biol 1999;11:36371

40 Ostareck-Lederer A, Ostareck DH, Standart N, et al. Translation of 15-lipoxygenase mRNA is inhibited by a protein that binds to a repeated sequence in the 3' untranslated region. EMBO J 1994;3:1476-81.

41 Ostareck DH, Ostareck-Lederer A, Wilm H, et al. mRNA silencing in erythroid differentiation: hnRNP $\mathrm{K}$ and hnRNP E1 regulate 15-lipoxygenase translation from the 3' end. Cell 1997;89:597-606.

42 Lee JC, Laydon JJ, McDonell PC, et al. A protein kinase involved in the regulation of inflammatory cytokine biosynthesis. Nature 1994;372:739-46.

43 Winzen R, Kracht M, Ritter B, et al. The p38 MAP kinase pathway signals for cytokine-induced mRNA stabilization via MAP kinase-activated protein kinase 2 and an AU-rich region-targeted mechanism. EMBO J 1999;18:4969-80.

44 Kotlyarov A, Neininger A, Schubert C, et al. MAPKAP kinase 2 is essential for LPS-induced TNF- $\alpha$ biosynthesis. Nature Cell Biology 1999;1:94-7.

45 Han J, Ulevitch RJ. Emerging targets for anti-inflammatory therapy. Nature Cell Biology 1999;1:E39-40. 